\title{
Study of dark matter in the extended BLMSSM
}

\author{
Jing-Jing Feng, ${ }^{1, *}$ Shu-Min Zhao, ${ }^{1, \dagger}$ Xing-Xing Dong, ${ }^{1}$ Zhong-Jun Yang, ${ }^{1}$ Hai-Bin Zhang, ${ }^{1}$ Fang Wang, ${ }^{2}$ and Tai-Fu Feng ${ }^{1, \$}$ \\ ${ }^{1}$ Department of Physics and Technology, Hebei University, Baoding 071002, China \\ ${ }^{2}$ College of Electronic Information Engineering, Hebei University, Baoding 071002, China
}

(Received 23 January 2019; published 24 May 2019)

\begin{abstract}
There is strong evidence for the existence of dark matter in some experiments at present. However, the question is that we do not have a reasonable explanation for dark matter in the framework of the Standard Model (SM) of particle physics. It is necessary to extend the SM in order to explain the dark matter. According to the current possible existence conditions of dark matter, we choose $\chi_{L}^{0}$ and $\tilde{Y}$ as candidates for dark matter in the EBLMSSM. We study the dominant annihilation processes in detail, including $\bar{\chi}_{L}^{0} \chi_{L}^{0}(\overline{\tilde{Y}} \tilde{Y}) \rightarrow \bar{l}^{I} l^{I}$ and $\bar{\chi}_{L}^{0} \chi_{L}^{0}(\overline{\tilde{Y}} \tilde{Y}) \rightarrow \bar{\nu}^{I} \nu^{I}$. We calculate their annihilation cross section $\sigma$ and relic density $\Omega_{D} h^{2}$. Then we analyze the limitations of dark matter relic density on the parameters of the EBLMSSM.
\end{abstract}

DOI: 10.1103/PhysRevD.99.095033

\section{INTRODUCTION}

Astronomers are convinced that there is amazing amount of dark matter in the universe by some astronomical observations and theoretical derivations. The earliest and perhaps the most convincing evidence of the existence of dark matter until today comes from observations of the rotation curves of galaxies [1]. It is the graph of circular velocities of stars and gas as a function of their distance from the Galactic center. This observation is inconsistent with our calculations using Newtonian dynamics. Therefore, it can be inferred that there is a lot of invisible matter in the universe, which is called dark matter (DM) [2-4]. If you want to know more evidence of the existence of dark matter, you can find them in Refs. [5-9]. Dark matter is widespread and abundant, and it accounts for about $23 \%$ of the universe, while the common baryon matter only accounts for about 4\% [10,11]. However, the standard model (SM) of the particle physics cannot provide a convincing explanation. So studying dark matter is a meaningful and interesting work to explore new physics beyond SM.

Although we do not know what dark matter is and how it exists, we can deduce some of its properties by analyzing and calculating data. First, dark matter particles have to be both electrically and color neutral. Second, it only

\footnotetext{
*fengjj_0726@163.com

†haosm@hbu.edu.cn

*fengtf@hbu.edu.cn
}

Published by the American Physical Society under the terms of the Creative Commons Attribution 4.0 International license. Further distribution of this work must maintain attribution to the author(s) and the published article's title, journal citation, and DOI. Funded by SCOAP. participates in weak interactions. Finally it must remain stable and have a long life, otherwise it will decay into other particles $[12,13]$. Only neutrinos in SM can have the correct interaction properties, but it is known that the masses of neutrinos are too small to constitute the main component of dark matter. Considering the above conditions, there are no suitable dark matter candidates in SM. It is certain that the existence of suitable dark matter particle is beyond SM. In recent years, weakly interacting massive particles (WIMPS) have become the most popular candidates for cold dark matter. Many experiments are detecting it, so experimental limitations continue to strengthen.

In fact, not only the problem of dark matter but also many other phenomena are not explained well by SM, and these problems and phenomena may become strong evidence for the exploration of new physics. The minimal supersymmetric extension of the standard model (MSSM) is a popular theory to explain these anomalies. Physicists in various countries have been studying it for many years. The BLMSSM is the simple extension of MSSM and the baryon and lepton numbers are local gauge symmetries spontaneously broken at the TeV scale in BLMSSM. Since the matter-antimatter is asymmetrical in the universe, the baryon number(B) is broken. On the other hand, considering the neutrino oscillation experiments, the heavy majorana neutrinos which are contained in the seesaw mechanism can induce the tiny neutrino masses. Therefore, the lepton number $(\mathrm{L})$ is also expected to be broken. The proton remains stable and R-parity is not conserved. Consequently the predictions and bounds for the collider experiments should be changed in the BLMSSM.

Although the BLMSSM can explain many anomalies, we find that the exotic leptons in the BLMSSM are not heavy enough. The diagonal elements of their mass matrix are zero. Therefore, the masses of the exotic leptons are 
only related to the four parameters $Y_{e 4}, Y_{e 5}, v_{d}$, and $v_{u}$. Here $v_{d}$ and $v_{u}$ are the vacuum expectation values (VEVs) of two Higgs doublets $H_{d}$ and $H_{u}$. They need to satisfy the equation $\sqrt{v_{d}^{2}+v_{u}^{2}}=v \sim 250 \mathrm{GeV}$. At the same time, the Yukawa couplings $Y_{e 4}$ and $Y_{e 5}$ are not large parameters. It can be calculated that the masses of the exotic leptons are approximately $100 \mathrm{GeV}$. With the development of high energy physics experiments, this boundary will be ruled out in the near future. It is related to whether the BLMSSM can continue to exist. Therefore, in order to get heavy exotic leptons, we add two exotic Higgs superfields which are $S U$ (2) singlets $\Phi_{N L}$ and $\varphi_{N L}$ with VEVs of $v_{N L}$ and $\bar{v}_{N L}$ to the BLMSSM. In this way, the mass matrix of the exotic leptons becomes Eq. (6). These diagonal elements can be large and contribute to mass, so the masses of exotic leptons become heavy enough. Because the heavy exotic leptons should be unstable, the two superfields $Y$ and $Y^{\prime}$ are introduced. On the other hand, the fourth and fifth generation leptons are mixed, which is different from the BLMSSM. It is obvious that the first four terms of $\mathcal{W}_{B}$ and $\mathcal{W}_{L}$ in Eq. (1) are exactly corresponding. We call this new model EBLMSSM which is an extension of the BLMSSM. Fortunately, we also find several new candidates for cold dark matter in the EBLMSSM. In our previous work, we have studied $Y$ as dark matter candidate in the EBLMSSM [14] and we study $\chi_{L}^{0}$ and $\tilde{Y}$ in this paper. Here, we study dark matter candidates annihilating into leptons and light neutrinos in the EBLMSSM.

The biggest clue is the observation of the relic density for dark matter in the universe, which is a strong constraint on the model to explain dark matter until now. The latest experimental observations show that the dark matter relic density is $0.1186 \pm 0.0020$ [15]. Furthermore, there are constraints on the mass of Higgs. In general, the EBLMSSM meet the above constraints. In our work, the DM relic density should satisfy $0.1186 \pm 0.0020$ within $3 \sigma$ range. It is strictly limited, and leads to that the parameters in the EBLMSSM can vary only in a narrow range. Our work can not only provide a possibility to explain dark matter, but also provide a direction for indirect detection experiments.

The remaining of the paper is organized as follows: In Sec. II we introduce the EBLMSSM model in detail. In Sec. III we discuss the relic density in the universe at present and we calculate annihilation cross section of dark matter candidates. Section IV is focused on the numerical analysis. In Sec. V, we give our discussion and conclusion.

\section{INTRODUCTION OF THE MODEL}

In this section, we briefly introduce the basic characteristics of EBLMSSM. It is the extension of BLMSSM. The local gauge group is $S U(3)_{C} \otimes S U(2)_{L} \otimes U(1)_{Y} \otimes$ $U(1)_{B} \otimes U(1)_{L}$ [16-19]. Compared with the BLMSSM, the EBLMSSM includes new superfields. In order to generate large masses for the exotic leptons, we need to introduce the two new superfields $\left(\Phi_{N L}\right.$ and $\left.\varphi_{N L}\right)$ with nonzero vacuum expectation values $\left(v_{N L}\right.$ and $\left.\bar{v}_{N L}\right)$. At the same time, the other two new superfields $\left(Y\right.$ and $\left.Y^{\prime}\right)$ are also added to keep the heavy exotic leptons unstable [14]. The superpotential of EBLMSSM is given by

$$
\begin{aligned}
\mathcal{W}_{\mathrm{EBLMSSM}} & =\mathcal{W}_{\mathrm{MSSM}}+\mathcal{W}_{B}+\mathcal{W}_{L}+\mathcal{W}_{X}+\mathcal{W}_{Y}, \\
\mathcal{W}_{B} & =\lambda_{Q} \hat{Q}_{4} \hat{Q}_{5}^{c} \hat{\varphi}_{B}+\lambda_{U} \hat{U}_{4}^{c} \hat{U}_{5} \hat{\Phi}_{B}+\lambda_{D} \hat{D}_{4}^{c} \hat{D}_{5} \hat{\Phi}_{B}+\mu_{B} \hat{\Phi}_{B} \hat{\varphi}_{B}+Y_{u_{4}} \hat{Q}_{4} \hat{H}_{u} \hat{U}_{4}^{c}+Y_{d_{4}} \hat{Q}_{4} \hat{H}_{d} \hat{D}_{4}^{c}+Y_{u_{5}} \hat{Q}_{5}^{c} \hat{H}_{d} \hat{U}_{5}+Y_{d_{5}} \hat{Q}_{5}^{c} \hat{H}_{u} \hat{D}_{5}, \\
\mathcal{W}_{L} & =\lambda_{L} \hat{L}_{4} \hat{L}_{5}^{c} \hat{\varphi}_{N L}+\lambda_{E} \hat{E}_{4}^{c} \hat{E}_{5} \hat{\Phi}_{N L}+\lambda_{N L} \hat{N}_{4}^{c} \hat{N}_{5} \hat{\Phi}_{N L}+\mu_{N L} \hat{\Phi}_{N L} \hat{\varphi}_{N L} \\
& +Y_{e_{4}} \hat{L}_{4} \hat{H}_{d} \hat{E}_{4}^{c}+Y_{\nu_{4}} \hat{L}_{4} \hat{H}_{u} \hat{N}_{4}^{c}+Y_{e_{5}} \hat{L}_{5}^{c} \hat{H}_{u} \hat{E}_{5}+Y_{\nu_{5}} \hat{L}_{5}^{c} \hat{H}_{d} \hat{N}_{5}+Y_{\nu} \hat{L} \hat{H}_{u} \hat{N}^{c}+\lambda_{N^{c}} \hat{N}^{c} \hat{N}^{c} \hat{\varphi}_{L}+\mu_{L} \hat{\Phi}_{L} \hat{\varphi}_{L}, \\
\mathcal{W}_{Y} & =\lambda_{4} \hat{L} \hat{L}_{5}^{c} \hat{Y}+\lambda_{5} \hat{N}^{c} \hat{N}_{5} \hat{Y}^{\prime}+\lambda_{6} \hat{E}^{c} \hat{E}_{5} \hat{Y}^{\prime}+\mu_{Y} \hat{Y} \hat{Y}^{\prime},
\end{aligned}
$$

where $\mathcal{W}_{\text {MSSM }}$ represents the superpotential of the MSSM. $\mathcal{W}_{B}$ and $\mathcal{W}_{X}$ are the same as the terms in the BLMSSM. $\mathcal{W}_{L}$ is different from BLMSSM for adding the first four items in Eq. (1) [20,21]. $\mathcal{W}_{Y}$ has some new couplings including the lepton-exotic lepton- $Y$ coupling and leptonexotic slepton- $\tilde{Y}$ coupling. Furthermore, we can also acquire lepton-slepton-lepton neutralino coupling. Here $\tilde{Y}$ is the superpartners of $Y$ and $Y^{\prime}$ and its four component spinor. In fact, the new couplings of $l^{I}-L^{\prime}-Y$ and $l^{I}-$ $\tilde{L}^{\prime}-\tilde{Y}$ have a great influence on lepton anormal magnetic dipole moment (MDM) in one loop order. They are able to correct the muon MDM and match the experimental values very well. New parameter $\mu_{Y}$ provides a new source for $C P$-violation. If $\lambda_{4}\left(\lambda_{6}\right)$ in Eq. (11) has nonzero elements about lepton flavor, $\mathcal{W}_{Y}$ can enhance the impact of lepton flavor violation [20]. In short, these new couplings and new parameters enrich the lepton physics to a certain degree. In addition, the study of dark matter has been promoted. It provides a new possibility for explaining dark matter. Besides the above mentioned problems, we can also study many other new physics problems in the EBLMSSM. Three particles $(\tilde{X}, X$, and $Y)$ as dark matter candidates have been discussed in previous work [14,17]. The particles $\left(X_{L}^{0}\right.$ and $\left.\tilde{Y}\right)$ will be discussed in this paper.

Based on the new introduced superfields $\Phi_{N L}, \varphi_{N L}, Y$, and $Y^{\prime}$ in the EBLMSSM, the soft breaking terms are as follows 


$$
\begin{aligned}
\mathcal{L}_{\text {soft }}^{\text {EBLMSSM }}= & \mathcal{L}_{\text {soft }}^{\text {BLMSSM }}-m_{\Phi_{N L}}^{2} \Phi_{N L}^{*} \Phi_{N L}-m_{\varphi_{N L}}^{2} \varphi_{N L}^{*} \varphi_{N L} \\
& +\left(A_{L L} \lambda_{L} \tilde{L}_{4} \tilde{L}_{5}^{c} \varphi_{N L}+A_{L E} \lambda_{E} \tilde{e}_{4}^{c} \tilde{e}_{5} \Phi_{N L}\right. \\
& \left.+A_{L N} \lambda_{N L} \tilde{\nu}_{4}^{c} \tilde{\nu}_{5} \Phi_{N L}+B_{N L} \mu_{N L} \Phi_{N L} \varphi_{N L}+\text { H.c. }\right) \\
& +\left(A_{4} \lambda_{4} \tilde{L} \tilde{L}_{5}^{c} Y+A_{5} \lambda_{5} \tilde{N}^{c} \tilde{\nu}_{5} Y^{\prime}+A_{6} \lambda_{6} \tilde{e}^{c} \tilde{e}_{5} Y^{\prime}\right. \\
& \left.+B_{Y} \mu_{Y} Y Y^{\prime}+\text { H.c. }\right) .
\end{aligned}
$$

$\mathcal{L}_{\text {soft }}^{\text {BLMSM }}$ is the soft breaking terms of the BLMSSM discussed in our previous work. $S U(2)_{L}$ singlets $\Phi_{L}$, $\varphi_{L}, \Phi_{N L}, \varphi_{N L}$ acquire the nonzero VEVs $v_{L}, \bar{v}_{L}, v_{N L}, \bar{v}_{N L}$, respectively. The local gauge symmetry $S U(2)_{L} \otimes$ $U(1)_{Y} \otimes U(1)_{B} \otimes U(1)_{L}$ breaks down to electromagnetic symmetry $U(1)_{e}$,

$$
\begin{aligned}
\Phi_{L} & =\frac{1}{\sqrt{2}}\left(v_{L}+\Phi_{L}^{0}+i P_{L}^{0}\right), \quad \varphi_{L}=\frac{1}{\sqrt{2}}\left(\bar{v}_{L}+\varphi_{L}^{0}+i \bar{P}_{L}^{0}\right), \\
\Phi_{N L} & =\frac{1}{\sqrt{2}}\left(v_{N L}+\Phi_{N L}^{0}+i P_{N L}^{0}\right) \\
\varphi_{N L} & =\frac{1}{\sqrt{2}}\left(\bar{v}_{N L}+\varphi_{N L}^{0}+i \bar{P}_{N L}^{0}\right)
\end{aligned}
$$

In the EBLMSSM, some mass matrices are different from BLMSSM because of the introduced superfields $\Phi_{N L}$ and $\varphi_{N L}$. We list some mass matrices and new couplings as following. If you want to know more of the mass matrixes and the couplings, you can find them in our previous work $[22,23]$.

\section{A. The mass matrices}

\section{The lepton neutralino mass matrix in the EBLMSSM}

In the EBLMSSM, $\lambda_{L}$, the superpartner of the new lepton type gauge boson $Z_{L}^{\mu}$, mixes with the SUSY superpartners $\left(\psi_{\Phi_{L}}, \psi_{\varphi_{L}}, \psi_{\Phi_{N L}}, \psi_{\varphi_{N L}}\right)$ of the superfields $\left(\Phi_{L}, \varphi_{L}, \Phi_{N L}, \varphi_{N L}\right)$. So the lepton neutralino mass matrix is obtained in the base $\left(i \lambda_{L}, \psi_{\Phi_{L}}, \psi_{\varphi_{L}}, \psi_{\Phi_{N L}}, \psi_{\varphi_{N L}}\right)$,

$\mathcal{M}_{L}=\left(\begin{array}{ccccc}2 M_{L} & 2 v_{L} g_{L} & -2 \bar{v}_{L} g_{L} & 3 v_{N L} g_{L} & -3 \bar{v}_{N L} g_{L} \\ 2 v_{L} g_{L} & 0 & -\mu_{L} & 0 & 0 \\ -2 \bar{v}_{L} g_{L} & -\mu_{L} & 0 & 0 & 0 \\ 3 v_{N L} g_{L} & 0 & 0 & 0 & -\mu_{N L} \\ -3 \bar{v}_{N L} g_{L} & 0 & 0 & -\mu_{N L} & 0\end{array}\right)$.

The mass matrix $\mathcal{M}_{L}$ can be diagonalized by the rotation matrix $Z_{N L}$. Then, we can have

$$
\begin{aligned}
& i \lambda_{L}=Z_{N L}^{1 i} K_{L_{i}}^{0}, \quad \psi_{\Phi_{L}}=Z_{N L}^{2 i} K_{L_{i}}^{0}, \quad \psi_{\varphi_{L}}=Z_{N L}^{3 i} K_{L_{i}}^{0}, \\
& \psi_{\Phi_{N L}}=Z_{N L}^{4 i} K_{L_{i}}^{0}, \quad \psi_{\varphi_{N L}}=Z_{N L}^{5 i} K_{L_{i}}^{0} .
\end{aligned}
$$

Here, $X_{L_{i}}^{0}=\left(K_{L_{i}}^{0}, \bar{K}_{L_{i}}^{0}\right)^{T}$ represent the mass eigenstates of the lepton neutralino.

\section{The exotic lepton mass matrix in the EBLMSSM}

The mass matrix for the exotic leptons reads as

$$
\begin{aligned}
-\mathcal{L}_{e^{\prime}}^{\text {mass }}= & \left(\bar{e}_{4 R}^{\prime}, \bar{e}_{5 R}^{\prime}\right)\left(\begin{array}{cc}
-\frac{1}{\sqrt{2}} \lambda_{L} \bar{v}_{L} & -\frac{1}{\sqrt{2}} Y_{e 5} v_{u} \\
-\frac{1}{\sqrt{2}} Y_{e 4} v_{d} & \frac{1}{\sqrt{2}} \lambda_{E} v_{L}
\end{array}\right) \\
& \times\left(\begin{array}{c}
e_{4 L}^{\prime} \\
e_{5 L}^{\prime}
\end{array}\right)+\text { H.c. }
\end{aligned}
$$

\section{The $\tilde{Y}$ mass matrix in the EBLMSSM}

The mass term for superfield $\tilde{Y}$ in the Lagrangian is given out

$$
-\mathcal{L}_{\tilde{Y}}^{\text {mass }}=\mu_{Y} \overline{\tilde{Y}} \tilde{Y}, \quad \tilde{Y}=\left(\begin{array}{c}
\psi_{Y^{\prime}} \\
\bar{\psi}_{Y}
\end{array}\right) .
$$

Here $m_{\tilde{Y}}($ the mass of $\tilde{Y})=\mu_{Y}$.

\section{B. Some couplings}

\section{The couplings with $\chi_{L}^{0}$}

As a dark matter candidate, lepton neutralinos $\left(\chi_{L}^{0}\right)$ not only have relations with leptons $(l)$ and sleptons $(\tilde{L})$, but also act with neutrinos $(\nu)$ and sneutrinos $(\tilde{\nu})$.

$$
\begin{aligned}
\mathcal{L}\left(\chi_{L}^{0} \tilde{L} l\right)= & \sum_{I=1}^{3} \sum_{i=1}^{6} \sqrt{2} g_{L} \bar{\chi}_{L_{j}}^{0}\left(Z_{N L}^{1 j} Z_{\tilde{L}}^{I i} P_{L}-Z_{N L}^{1 j *} Z_{\tilde{L}}^{(I+3) i} P_{R}\right) l^{I} \tilde{L}_{i}^{+} \\
& + \text {H.c. } \\
\mathcal{L}\left(\chi_{L}^{0} \tilde{\nu} \nu\right)= & \sum_{I, J=1}^{3} \sum_{\alpha, j=1}^{6} \bar{X}_{N_{\alpha}}\left(\left[-\left(\lambda_{N_{c}}^{I J}+\lambda_{N_{c}}^{J I}\right) Z_{N_{\nu}}^{(I+3) \alpha} Z_{N_{L}}^{3 i} Z_{\tilde{\nu}}^{(J+3) j *}\right.\right. \\
& \left.+\sqrt{2} g_{L} Z_{N_{\nu}}^{I \alpha} Z_{N_{L}}^{1 i} Z_{\tilde{\nu}}^{J j *} \delta_{I J}\right] P_{L} \\
& \left.\left.-\sqrt{2} g_{L} Z_{N_{\nu}}^{(I+3) \alpha *} Z_{N_{L}}^{1 i *} Z_{\tilde{\nu}}^{(J+3) j *} \delta_{I J}\right] P_{R}\right) X_{L_{i}}^{0} \tilde{\nu}^{j *}+\text { H.c. }
\end{aligned}
$$

The coupling for lepton neutralino-new gauge boson $Z_{L^{-}}^{\mu}$ lepton neutralino reads as

$$
\begin{aligned}
\mathcal{L}\left(X_{L}^{0} X_{L}^{0} Z_{L}^{\mu}\right)= & -g_{L} Z_{L}^{\mu} \bar{X}_{L_{i}}^{0} \gamma_{\mu}\left(3 Z_{N_{L}}^{5 i *} Z_{N_{L}}^{5 j}-3 Z_{N_{L}}^{4 *} Z_{N_{L}}^{4 j}\right) P_{L} X_{L_{j}}^{0} \\
& + \text { H.c. }
\end{aligned}
$$

\section{The couplings with $\tilde{Y}$}

For the dark matter candidate $\tilde{Y}$, in addition to the interactions with exotic sleptons $\left(\tilde{L}^{\prime}\right)$ and leptons $(l)$, there are also interactions with exotic sneutrinos $\left(\tilde{N}^{\prime}\right)$ and neutrinos $(\nu)$, whose couplings are in the following form 


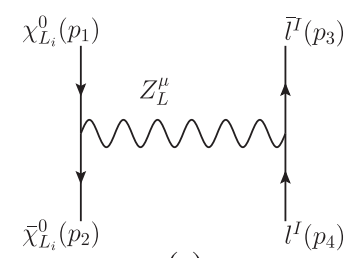

(a)

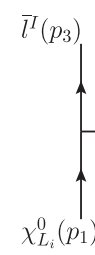

$\chi_{L_{i}}\left(p_{1}\right)$ (b)

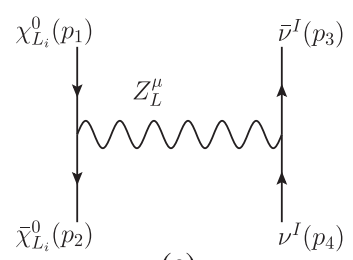

(c)

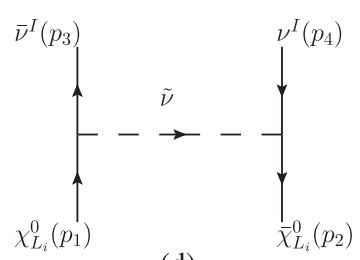

(d)

FIG. 1. Feynman diagrams for the $\chi_{L_{i}}^{0} \chi_{L_{i}}^{0} \rightarrow Z_{L}^{\mu} \rightarrow \bar{l}^{I} l^{I}\left(\bar{\nu}^{I} \nu^{I}\right)$ and $\chi_{L_{i}}^{0} \chi_{L_{i}}^{0} \rightarrow \tilde{L}(\tilde{\nu}) \rightarrow \bar{l}^{I} l^{I}\left(\bar{\nu}^{I} \nu^{I}\right)$ at the tree level.

$\mathcal{L}\left(\tilde{Y} l \tilde{L^{\prime}}\right)=\sum_{I=1}^{3} \sum_{i=1}^{4} \overline{\tilde{Y}}\left(-\lambda_{4} Z_{\tilde{L}^{\prime}}^{4 i *} P_{L}-\lambda_{6} Z_{\tilde{L}^{\prime}}^{3 i *} P_{R}\right) e^{I} \tilde{L}_{i}^{\prime *}+$ H.c.

$$
\begin{aligned}
\mathcal{L}\left(\tilde{Y} \nu \tilde{N}^{\prime}\right)= & \sum_{I=1}^{3} \sum_{i=1}^{4} \sum_{\alpha=1}^{6} \overline{\tilde{Y}}\left(-\lambda_{4} Z_{N_{\nu}}^{I \alpha} Z_{\tilde{N}^{\prime}}^{4 i *} P_{L}\right. \\
& \left.-\lambda_{5} Z_{N_{\nu}}^{(I+3) \alpha *} Z_{\tilde{N}^{\prime}}^{3 i *} P_{R}\right) X_{N_{\alpha}}^{0} \tilde{N}_{i}^{\prime *}+\text { H.c. }
\end{aligned}
$$

$\tilde{Y}$ interacts with $Z_{L}^{\mu}$ and $\tilde{Y}$, whose coupling is in the following form

$$
\mathcal{L}\left(\tilde{Y} \tilde{Y} Z_{L}^{\mu}\right)=\overline{\tilde{Y}}\left[g_{L}\left(2+L_{4}\right) \gamma_{\mu}\right] Z_{L}^{\mu} \tilde{Y}+\text { H.c. }
$$

The new gauge boson $Z_{L}^{\mu}$ couples with leptons and neutrinos, whose couplings can be find in our previous work [14].

\section{DARK MATTER CANDIDATES: $X_{L}^{0}$ AND $\tilde{Y}$}

In this section, we suppose the lightest mass eigenstate of $X_{L}^{0}$ and $\tilde{Y}$ as dark matter candidates. They belong to weakly-interacting massive particles (WIMPs) that are the most studied dark matter candidates. The mass range of WIMPs is $10 \mathrm{GeV}-\mathrm{TeV}$. They interact with the $W$ and $Z$ gauge bosons, but not with photons at tree level [1,24-26]. So we summarize the relic density constraint that any WIMP candidates should satisfy. First, we need to introduce the freeze-out temperature $T_{F}$. And $T_{F}$ is usually expressed as a dimensionless quantity $x=\frac{m_{D}}{T_{F}}$, where $m_{D}$ is dark matter mass. Then, we get the concrete form of $x_{F}$ by solving the Boltzmann equation of the dark matter number density $n$ [27-30].

$$
\dot{n}=-3 H n-\langle\sigma v\rangle\left(n^{2}-n_{0}^{2}\right)
$$

Here $n$ is the number density of the dark matter, $\sigma$ is the annihilation cross section of the particle, $v$ is the relative velocity of the annihilating particles, $H$ is the expansion rate of the Universe and $n_{0}$ is the dark matter number density in thermally equilibrium. Finally, we can obtain an iterative equation about $x_{F}$ for solving Eq. (14).

$$
x_{F}=\ln \frac{0.076 M_{\mathrm{pl}} m_{D}\langle\sigma v\rangle}{\sqrt{g_{*} x_{F}}} .
$$

Here, $M_{\mathrm{pl}}=1.22 \times 10^{19} \mathrm{GeV}$ is the Planck mass and $g_{*}$ is the number of the relativistic degrees of freedom with mass less than $T_{F}$. We can calculate cross section and the term $\langle\sigma v\rangle$ in the Eqs. (14)-(15) can be written as

$$
\langle\sigma v\rangle=a+b v^{2}+\mathcal{O}\left(v^{4}\right)
$$

$a$ and $b$ are the first two coefficients in the Taylor expansion of the annihilation cross section [14,28,31]. We use the formula of the present relic density $\left(\Omega_{D} h^{2}\right)$ of DM candidates, assuming that the mass $m_{D}$ as well as the annihilation cross section $\sigma$ are known. Furthermore, neglecting the terms at the order of $\mathcal{O}\left(v^{4}\right)$, we give the expression of $x_{F}$ and calculate $\Omega_{D} h^{2}$ [32-34] by

$$
\Omega_{D} h^{2} \simeq \frac{1.07 \times 10^{9} x_{F}}{\sqrt{g_{*}} M_{\mathrm{pl}}\left(a+3 b / x_{F}\right) \mathrm{GeV}}
$$

\section{A. $a$ and $b$ of $\chi_{L}^{0}$}

We show the most important lepton neutralino $\left(\chi_{L}^{0}\right)$ annihilation diagrams whose final states are leptons and light neutrinos. For all the tree-level processes (in Fig. 1), we can calculate $a$ and $b$ (in the low velocity limit) by using the couplings in Eqs. (8)-(10).

$X_{1}=Z_{N_{L}}^{5 i *} Z_{N_{L}}^{5 i}-Z_{N_{L}}^{4 i *} Z_{N_{L}}^{4 i}, \quad X_{2}=Z_{N_{L}}^{1 i} Z_{\tilde{L}}^{I j}+Z_{N_{L}}^{1 i *} Z_{\tilde{L}}^{(I+3) j}$,

$X_{3}=Z_{N_{L}}^{1 i} Z_{\tilde{L}}^{I j}-Z_{N_{L}}^{1 i *} Z_{\tilde{L}}^{(I+3) j}, \quad X_{1}^{\prime}=\frac{1}{\sqrt{2}} X_{1}$,

$X_{2}^{\prime}=X_{3}^{\prime}=Z_{N_{L}}^{1 i} Z_{\tilde{\nu}}^{J j *}, \quad A_{1}=\left|X_{2}\right|^{2}+\left|X_{3}\right|^{2}$,

$A_{2}=-\left|X_{2}\right|^{2}+\left|X_{3}\right|^{2}, \quad A_{3}=\left|X_{2}\right|^{2}-\left|X_{3}\right|^{2}$,

$A_{4}=X_{2} X_{3}^{*}+X_{2}^{*} X_{3}, \quad A_{1}^{\prime}=\left|X_{2}^{\prime}\right|^{2}+\left|X_{3}^{\prime}\right|^{2}=2\left|X_{2}^{\prime}\right|^{2}$,

$A_{2}^{\prime}=A_{3}^{\prime}=0, \quad A_{4}^{\prime}=X_{2}^{\prime} X_{3}^{\prime *}+X_{2}^{\prime *} X_{3}^{\prime}=2\left|X_{2}^{\prime}\right|^{2}$.

We define the above terms to simplify the following formulas. They are all expressions relating to couplings. Next we write down the concrete expression of $a$ and $b$. 


$$
\begin{aligned}
& a=\sum_{l=e, \mu, \tau}\left\{\frac{g_{L}^{4}}{8 \pi}\left(1+\frac{m_{l}^{2}}{m_{D}^{2}}\right)^{\frac{1}{2}}\left[\frac{9\left(2 m_{D}^{2}+m_{l}^{2}\right)}{\left(4 m_{D}^{2}-m_{Z_{L}}^{2}\right)^{2}}\left|X_{1}\right|^{2}+\frac{\left(m_{D} A_{1}+m_{l} A_{3}\right)^{2}}{4\left(m_{D}^{2}-m_{l}^{2}+m_{\tilde{L}}^{2}\right)^{2}}+\sum_{j=1}^{6} \frac{3\left(2 m_{D}^{2}+m_{l}^{2}\right) A_{1}-3 m_{D} m_{l} A_{2}+6 m_{D} m_{l} A_{3}}{2\left(4 m_{D}^{2}-m_{Z_{L}}^{2}\right)\left(m_{D}^{2}-m_{l}^{2}+m_{\tilde{L}_{j}}^{2}\right)} X_{1}^{*}\right]\right\} \\
& +\sum_{\chi_{N_{\alpha}}^{0}=\nu_{e}, \nu_{\mu}, \nu_{\tau}}\left\{\frac { g _ { L } ^ { 4 } m _ { D } ^ { 2 } } { 8 \pi } \left[\frac{18}{\left(4 m_{D}^{2}-m_{Z_{L}}^{2}\right)^{2}}\left|X_{1}^{\prime}\right|^{2}+\frac{1}{\left(m_{D}^{2}+m_{\tilde{\nu}}^{2}\right)^{2}}\left|X_{2}^{\prime}\right|^{4}\right.\right. \\
& \left.\left.-\frac{6 \sqrt{2}}{\left(4 m_{D}^{2}-m_{Z_{L}}^{2}\right)\left(m_{D}^{2}+m_{\tilde{\nu}}^{2}\right)} X_{1}^{\prime *}\left|X_{3}^{\prime}\right|^{2}\right]\right\} \\
& b=\sum_{l=e, \mu, \tau}\left\{\frac { g _ { L } ^ { 4 } } { 1 6 \pi } \left[\left(\frac{18 m_{D}^{2}}{\left(4 m_{D}^{2}-m_{Z_{L}}^{2}\right)^{2}}+\frac{12 m_{D}^{4}-21 m_{D}^{2} m_{Z_{L}}^{2}}{\left(4 m_{D}^{2}-m_{Z_{L}}^{2}\right)^{3}}\right)\left|X_{1}\right|^{2}+\left(\frac{m_{D}^{2}}{4\left(m_{D}^{2}+m_{\tilde{L}}^{2}\right)^{2}}\right.\right.\right. \\
& \left.+\frac{15 m_{D}^{6}+10 m_{D}^{4} m_{\tilde{L}}^{2}+7 m_{D}^{2} m_{\tilde{L}}^{4}}{12\left(m_{D}^{2}+m_{\tilde{L}}^{2}\right)^{4}}\right) A_{1}^{2}+\sqrt{2} X_{1}^{*} A_{2}\left(\frac{3 m_{D} m_{l}}{2\left(4 m_{D}^{2}-m_{Z_{L}}^{2}\right)\left(m_{D}^{2}+m_{\tilde{L}}^{2}\right)}\right. \\
& \left.+\frac{-3 m_{D}^{5} m_{l} m_{Z_{L}}^{2}-16 m_{D}^{5} m_{l} m_{\tilde{L}}^{2}-2 m_{D}^{3} m_{l} m_{Z_{L}}^{2} m_{\tilde{L}}^{2}-3 m_{D} m_{l} m_{Z_{L}}^{2} m_{\tilde{L}}^{4}}{4\left(4 m_{D}^{2}-m_{Z_{L}}^{2}\right)^{2}\left(m_{D}^{2}+m_{\tilde{L}}^{2}\right)^{3}}\right) \\
& +\left(\frac{m_{D}^{2}\left(-5 A_{1}+2 A_{4}\right)-m_{D} m_{l} A_{3}}{2\left(4 m_{D}^{2}-m_{Z_{L}}^{2}\right)\left(m_{D}^{2}+m_{\tilde{L}}^{2}\right)}+\frac{-m_{D}^{2}\left(m_{D}^{2}\left(A_{1}-A_{4}\right)-m_{D} m_{l} A_{3}\right)}{\left(4 m_{D}^{2}-m_{Z_{L}}^{2}\right)\left(m_{D}^{2}+m_{\tilde{L}}^{2}\right)^{2}}\right) \sqrt{2} X_{1}^{*} \\
& +\left(\frac{7 m_{D}^{5} m_{l}-2 m_{D}^{3} m_{l} m_{\tilde{L}}^{2}+3 m_{D} m_{l} m_{\tilde{L}}^{4}}{12\left(m_{D}^{2}+m_{\tilde{L}}^{2}\right)^{4}}+\frac{m_{D} m_{l}}{2\left(m_{D}^{2}+m_{\tilde{L}}^{2}\right)^{2}}\right) A_{1} A_{3} \\
& +\sqrt{2}\left(m_{D}^{2} X_{1}^{*} A_{1}+m_{D} m_{l} X_{1}^{*} A_{3}\right)\left(-\frac{3 m_{D} m_{l}}{\left(4 m_{D}^{2}-m_{Z_{L}}^{2}\right)\left(m_{D}^{2}+m_{\tilde{L}}^{2}\right)}\right. \\
& \left.\left.\left.+\frac{m_{D}^{2}\left(10 m_{D}^{4}-m_{D}^{2} m_{Z_{L}}^{2}+24 m_{D}^{2} m_{\tilde{L}}^{2}-3 m_{Z_{L}}^{2} m_{\tilde{L}}^{2}+6 m_{\tilde{L}}^{4}\right)}{\left(4 m_{D}^{2}-m_{Z_{L}}^{2}\right)^{2}\left(m_{D}^{2}+m_{\tilde{L}}^{2}\right)^{3}}\right)\right]\right\} \\
& +\sum_{\chi_{N_{\alpha}}^{0}=\nu_{e}, \nu_{\mu}, \nu_{\tau}}\left\{\left(m_{l} \rightarrow m_{\nu} \simeq 0, m_{\tilde{L}} \rightarrow m_{\tilde{\nu}}, X_{1} \rightarrow X_{1}^{\prime}, A_{k} \rightarrow A_{k}^{\prime}(k=1,2,3,4)\right\} .\right.
\end{aligned}
$$

Because the mass of the light neutrino is too small, we regard it as zero. When we compute the term $b$, we find the specific form of $b$ is tedious and complicated. To simplify the results, we perform a Taylor expansion on $\frac{m_{l}}{m_{D}}$ and retain it to the second order.

\section{B. $a$ and $b$ of $\tilde{Y}$}

Similarly, we can also calculate the results of $a$ and $b$ for $\tilde{Y}$. We give the dominant contribution to the annihilation cross section come from $\bar{l}^{I} l^{I}$ and $\bar{\nu}^{I} \nu^{I}$. The tree diagrams are shown in Fig. 2. To simplify the results, we use the following assumptions:

$$
\begin{aligned}
& Y_{1}=2+L_{4}, \quad Y_{2}=\lambda_{4} Z_{\tilde{L}^{\prime}}^{4 i *}-\lambda_{6} Z_{\tilde{L}^{\prime}}^{3 i *}, \quad Y_{3}=-\lambda_{4} Z_{\tilde{E}}^{4 i *}-\lambda_{6} Z_{\tilde{E}}^{3 i *}, \quad Y_{1}^{\prime}=Y_{1}, \quad Y_{2}^{\prime}=\lambda_{4} Z_{\tilde{N}^{\prime}}^{4 i *}, \\
& B_{1}=\left|Y_{2}\right|^{2}+\left|Y_{3}\right|^{2}, \quad B_{2}=-\left|Y_{2}\right|^{2}+\left|Y_{3}\right|^{2}, \quad B_{3}=\left|Y_{2}\right|^{2}-\left|Y_{3}\right|^{2} .
\end{aligned}
$$

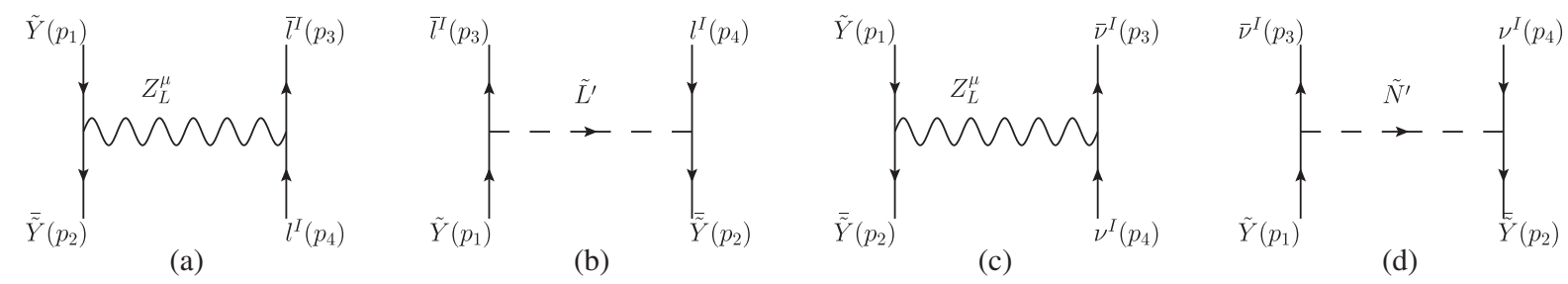

FIG. 2. Feynman diagrams for the $\tilde{Y} \tilde{Y} \rightarrow Z_{L}^{\mu} \rightarrow \bar{l}^{I} l^{I}\left(\bar{\nu}^{I} \nu^{I}\right)$ and $\tilde{Y} \tilde{Y} \rightarrow \tilde{L}^{\prime}\left(\tilde{N}^{\prime}\right) \rightarrow \bar{l}^{I} l^{I}\left(\bar{\nu}^{I} \nu^{I}\right)$ at the tree level. 
Using the couplings in Eqs. (11)-(13) and the expressions in Eq. (21), we deduce the results of $a$ and $b$.

$$
\begin{aligned}
& a=\sum_{l=e, \mu, \tau}\left\{\frac{g_{L}^{2}}{8 \pi}\left(1+\frac{m_{l}^{2}}{m_{D}^{2}}\right)^{\frac{1}{2}}\left[\frac{4 g_{L}^{2}\left(2 m_{D}^{2}+m_{l}^{2}\right)}{\left(4 m_{D}^{2}-m_{Z_{L}}^{2}\right)^{2}}\left|Y_{1}\right|^{2}+\frac{\left(m_{D} B_{1}+m_{l} B_{3}\right)^{2}}{16 g_{L}^{2}\left(m_{D}^{2}-m_{l}^{2}+m_{\tilde{L}^{\prime}}^{2}\right)^{2}}+\frac{\left(2 m_{D}^{2}+m_{l}^{2}\right) B_{1}-m_{D} m_{l} B_{2}+2 m_{D} m_{l} B_{3}}{2\left(4 m_{D}^{2}-m_{Z_{L}}^{2}\right)\left(m_{D}^{2}-m_{l}^{2}+m_{\tilde{L}^{\prime}}^{2}\right)} Y_{1}^{*}\right]\right\} \\
& +\sum_{\chi_{N_{\alpha}}^{0}=\nu_{e}, \nu_{\mu}, \nu_{\tau}}\left\{\frac{g_{L}^{2} m_{D}^{2}}{8 \pi}\left[\frac{4 g_{L}^{2}}{\left(4 m_{D}^{2}-m_{Z_{L}}^{2}\right)^{2}}\left|Y_{1}^{\prime}\right|^{2}+\frac{1}{4 g_{L}^{2}\left(m_{D}^{2}+m_{\tilde{N}^{\prime}}^{2}\right)^{2}}\left|Y_{2}^{\prime}\right|^{4}\right]\right\} \\
& b=\sum_{l=e, \mu, \tau}\left\{\frac { g _ { L } ^ { 2 } } { 1 6 \pi } \left[\left(\frac{8 m_{D}^{2} g_{L}^{2}}{\left(4 m_{D}^{2}-m_{Z_{L}}^{2}\right)^{2}}+\frac{4\left(-2 m_{D}^{4}-5 m_{D}^{2} m_{Z_{L}}^{2}\right) g_{L}^{2}}{3\left(4 m_{D}^{2}-m_{Z_{L}}^{2}\right)^{3}}\right)\left|Y_{1}\right|^{2}+\left(\frac{m_{D}^{2}}{4 g_{L}^{2}\left(m_{D}^{2}+m_{\tilde{L}^{\prime}}^{2}\right)^{2}}+\frac{15 m_{D}^{6}+10 m_{D}^{4} m_{\tilde{L}^{\prime}}^{2}+7 m_{D}^{2} m_{\tilde{L}^{\prime}}^{4}}{12 g_{L}^{2}\left(m_{D}^{2}+m_{\tilde{L}^{\prime}}^{2}\right)^{4}}\right) B_{1}^{2}\right.\right. \\
& +B_{1} B_{3}\left(\frac{7 m_{D}^{5} m_{l}-2 m_{D}^{3} m_{l} m_{\tilde{L}^{\prime}}^{2}+3 m_{D} m_{l} m_{\tilde{L}^{\prime}}^{4}}{12 g_{L}^{2}\left(m_{D}^{2}+m_{\tilde{L}^{\prime}}^{2}\right)^{4}}+\frac{m_{D} m_{l}}{2 g_{L}^{2}\left(m_{D}^{2}+m_{\tilde{L}^{\prime}}^{2}\right)^{2}}\right) \\
& +\left(\frac{m_{D}^{2}\left(10 m_{D}^{4}-m_{D}^{2} m_{Z_{L}}^{2}+24 m_{D}^{2} m_{\tilde{L}^{\prime}}^{2}-3 m_{Z_{L}}^{2} m_{\tilde{L}^{\prime}}^{2}+6 m_{\tilde{L}^{\prime}}^{4}\right)}{3\left(4 m_{D}^{2}-m_{Z_{L}}^{2}\right)^{2}\left(m_{D}^{2}+m_{\tilde{L}^{\prime}}^{2}\right)^{3}}-\frac{1}{\left(4 m_{D}^{2}-m_{Z_{L}}^{2}\right)\left(m_{D}^{2}+m_{\tilde{L}^{\prime}}^{2}\right)}\right)\left(m_{D}^{2} B_{1}+m_{D} m_{l} B_{3}\right) \\
& +\left(\frac{-5 m_{D}^{2} B_{1}-3 m_{D} m_{l} B_{3}}{6\left(4 m_{D}^{2}-m_{Z_{L}}^{2}\right)\left(m_{D}^{2}+m_{\tilde{L}^{\prime}}^{2}\right)}-\frac{m_{D}^{4} B_{1}-m_{D}^{2} B_{3}}{3\left(4 m_{D}^{2}-m_{Z_{L}}^{2}\right)\left(m_{D}^{2}+m_{\tilde{L}^{\prime}}^{2}\right)^{2}}\right) Y_{1}^{*} \\
& \left.\left.+Y_{1}^{*} B_{2}\left(\frac{m_{D} m_{l}}{2\left(4 m_{D}^{2}-m_{Z_{L}}^{2}\right)\left(m_{D}^{2}+m_{\tilde{L}^{\prime}}^{2}\right)}+\frac{-3 m_{D}^{5} m_{l} m_{Z_{L}}^{2}-16 m_{D}^{5} m_{l} m_{\tilde{L}^{\prime}}^{2}-2 m_{D}^{3} m_{l} m_{Z_{L}}^{2} m_{\tilde{L}^{\prime}}^{2}-3 m_{D} m_{l} m_{Z_{L}}^{2} m_{\tilde{L}^{\prime}}^{4}}{12\left(4 m_{D}^{2}-m_{Z_{L}}^{2}\right)^{2}\left(m_{D}^{2}+m_{\tilde{L}^{\prime}}^{2}\right)^{3}}\right)\right]\right\} \\
& +\sum_{\chi_{N_{\alpha}}^{0}=\nu_{e}, \nu_{\mu}, \nu_{\tau}}\left\{\frac{m_{D}^{2}}{16 \pi}\left[\left(\frac{15 m_{D}^{4}+10 m_{D}^{2} m_{\tilde{N}^{\prime}}^{2}+7 m_{\tilde{N}^{\prime}}^{4}}{12\left(m_{D}^{2}+m_{\tilde{N}^{\prime}}^{2}\right)^{4}}+\frac{m_{D}^{2}}{4\left(m_{D}^{2}+m_{\tilde{N}^{\prime}}^{2}\right)}\right)\left|Y_{2}^{\prime}\right|^{4}+\left(\frac{4 g_{L}^{2}}{\left(4 m_{D}^{2}-m_{Z_{L}}^{2}\right)^{2}}+\frac{g_{L}^{2}\left(-4 m_{D}^{2}-5 m_{Z_{L}}^{2}\right)}{3\left(4 m_{D}^{2}-m_{Z_{L}}^{2}\right)^{3}}\right) Y_{1}^{\prime *}\right]\right\} .
\end{aligned}
$$

The mass rotation matrices corresponding to $\chi_{L}^{0}, \tilde{L}, \tilde{\nu}, \tilde{L}^{\prime}$, and $\tilde{N}^{\prime}$ are $Z_{N_{L}}, Z_{\tilde{L}}, Z_{\tilde{\nu}}, Z_{\tilde{L}^{\prime}}$, and $Z_{\tilde{N}^{\prime}}$.

\section{NUMERICAL RESULTS}

In this section, we calculate the numerical results. Current data imply that dark matter is five times more than normal matter and accounts for about a quarter of the universe. In Sec. I, we give precisely the constraint of the relic density of cold nonbaryonic dark matter and it is $\Omega_{D} h^{2}=0.1186 \pm$ 0.0020 [15]. Next we will discuss $\Omega h^{2}$ of the $\chi_{L}^{0}$ and $\tilde{Y}$.

\section{A. Numerical result of $\chi_{L}^{0}$}

To obtain more transparent numerical results, we adopt the following assumptions on parameter space:

$$
\begin{aligned}
& g_{L}=1 / 6, \quad \mu=0.5 \mathrm{TeV}, \quad v_{N l t}=3 \mathrm{TeV}, \\
& \tan \beta=10, \quad\left(\lambda_{N^{c}}\right)_{i i}=1, \quad \tan \beta_{L}=\tan \beta_{N L}=2, \\
& \left(A_{l}\right)_{i j}=\left(A_{l^{\prime}}\right)_{i j}=0(i \neq j), \quad\left(A_{l}\right)_{i i}=2 \mathrm{TeV}, \\
& \left(A_{l^{\prime}}\right)_{i i}=0.3 \mathrm{TeV}, \quad\left(M_{\tilde{L}}^{2}\right)_{i i}=4 \mathrm{TeV}^{2}, \\
& \left(M_{\tilde{L}}^{2}\right)_{i j}=0.1 \mathrm{TeV}^{2}(i \neq j) \text {. }
\end{aligned}
$$

Here $i=1,2,3, \mu_{L}=\mu_{N L}=0.8 \mathrm{TeV}$. The lightest $\chi_{L}^{0}$ $\operatorname{mass}\left(m_{\chi_{L}^{0}}\right)$ is denoted by $m_{D}, m_{\chi_{L}^{0}}=m_{D}$. In order to constrain the parameter space in EBLMSSM we need to satisfy experimental results of $\Omega_{D} h^{2}$. We study relic density $\Omega_{D} h^{2}$ and $x_{F}$ versus $M_{L}$ in Fig. 3. The grey area is the experimental results of $\Omega_{D} h^{2}$ in $3 \sigma$ and the solid line represents relationship between $\Omega_{D} h^{2}$ and $M_{L}$. Parameter $M_{L}$ presents in the diagonal parts of the $\chi_{L}^{0}$ mass matrix. Because the increase of $M_{L}$ leads to the decrease of $\langle\sigma v\rangle$, $\Omega_{D} h^{2}$ increases with the increase of $M_{L}$. Furthermore, parameter $M_{L}$ has a very large impact on $\Omega_{D} h^{2}$. The sensitive parameter $M_{L}$ is limited to $908-929 \mathrm{GeV}$ in order to satisfy the experiment bounds of $\Omega_{D} h^{2}$. At this time, $m_{D}$ is limited to about $300 \mathrm{GeV}$. On the other hand, the range of variation is very narrow for $x_{F}$, about 0.5 . The curve of $x_{F}$ increases as $M_{L}$ decreases, which is a negative correlation.

The strong impact of the parameter $\mu_{N L}$ on the $\Omega_{D} h^{2}$ is further illustrated by Fig. 4. $\mu_{N L}$ is related to the nondiagonal parts of the $\chi_{L}^{0}$ mass matrix in the EBLMSSM. In addition to the parameters in Eq. (24), we also suppose $M_{L}=2 \mathrm{TeV}$ and $\mu_{L}=1.1 \mathrm{TeV}$. As can be seen from Fig. 4, when $\mu_{N L}$ increases, $\Omega_{D} h^{2}$ shows a downward trend. Different from the diagonal element $M_{L}$, the nondiagonal element $\mu_{N L}$ causes $\langle\sigma v\rangle$ increases. As sensitive parameter, $\mu_{N L}$ is limited in the narrow range of $1248-1256 \mathrm{GeV}$ when $\Omega_{D} h^{2}$ satisfies the experiment bounds. Its range is about half of the parameter $M_{L}$. Besides, the curve of $x_{F}$ is very 

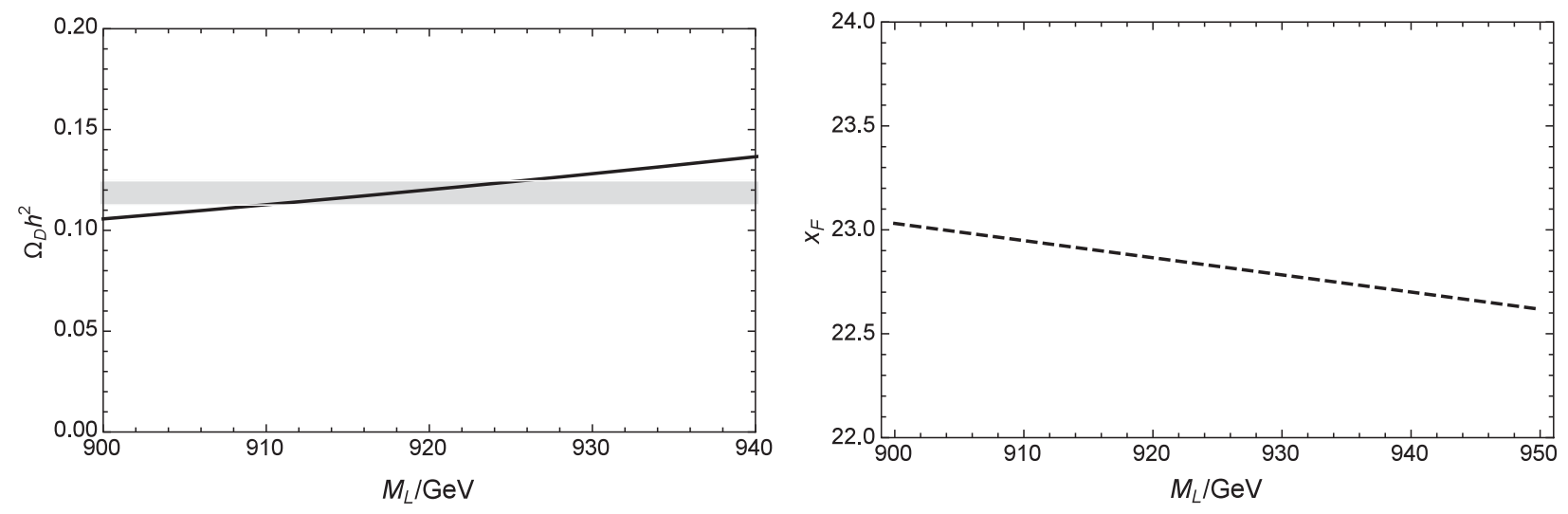

FIG. 3. The relic density and $x_{F}$ versus $M_{L}$ for $\chi_{L}^{0}$.
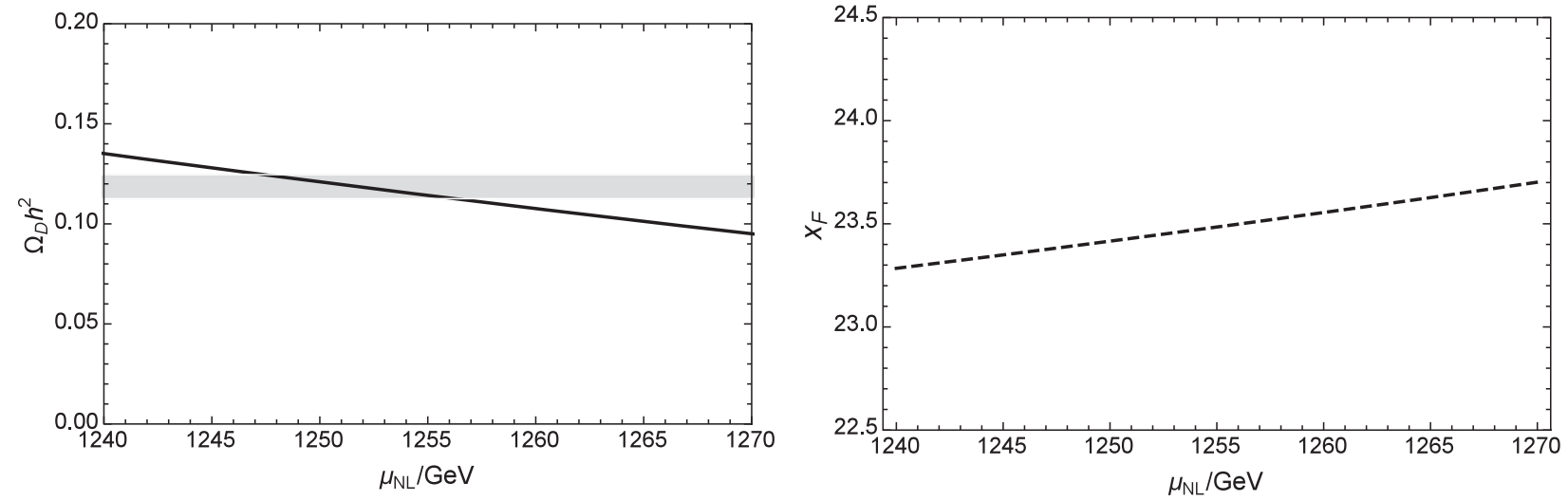

FIG. 4. The relic density and $x_{F}$ versus $\mu_{N L}$ for $\chi_{L}^{0}$.

slowly rising. $x_{F}$ is related to $\langle\sigma v\rangle$ and increases as $\langle\sigma v\rangle$ increases.

\section{B. Numerical result of $\tilde{Y}$}

To obtain the numerical results, we adopt the following parameters as

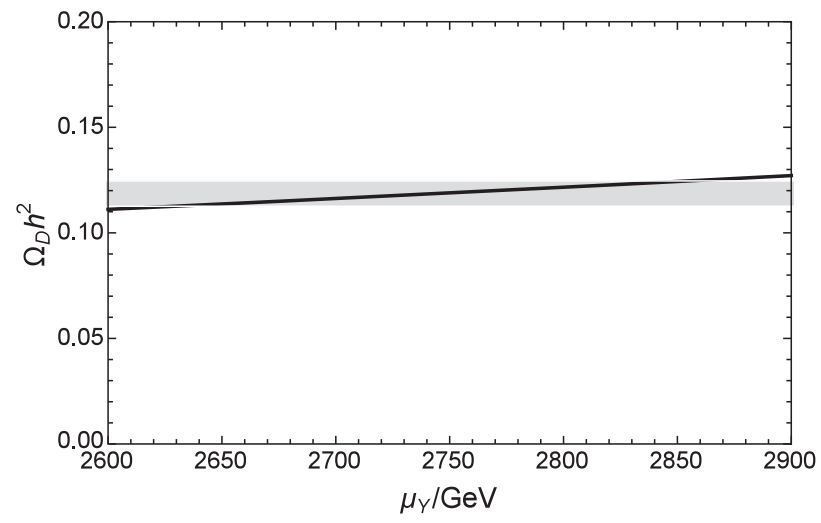

$$
\begin{aligned}
L_{4} & =1.5, \quad Y_{e 5}=1.2, \quad \mu=0.5 \mathrm{TeV}, \quad Y_{\nu 4}=Y_{\nu 5}=1.2, \\
\mu_{N L} & =1 \mathrm{TeV}, \quad \lambda_{E}=\lambda_{L}=\lambda_{N L}=\lambda^{\prime}, \quad \lambda_{4}=\lambda_{5}=\lambda_{6}=L_{m}, \\
A_{e_{4}} & =A_{e_{5}}=A_{\nu_{4}}=A_{\nu_{5}}=1 \mathrm{TeV}, \quad A_{L L}=A_{L E}=A_{L N}=1 \mathrm{TeV}, \\
M_{\tilde{L}_{4}}^{2} & =M_{\tilde{L}_{5}}^{2}=M_{\tilde{e}_{4}}^{2}=M_{\tilde{e}_{5}}^{2}=M_{\tilde{\nu}_{4}}^{2}=M_{\tilde{\nu}_{5}}^{2}=1 \mathrm{TeV}^{2} .
\end{aligned}
$$

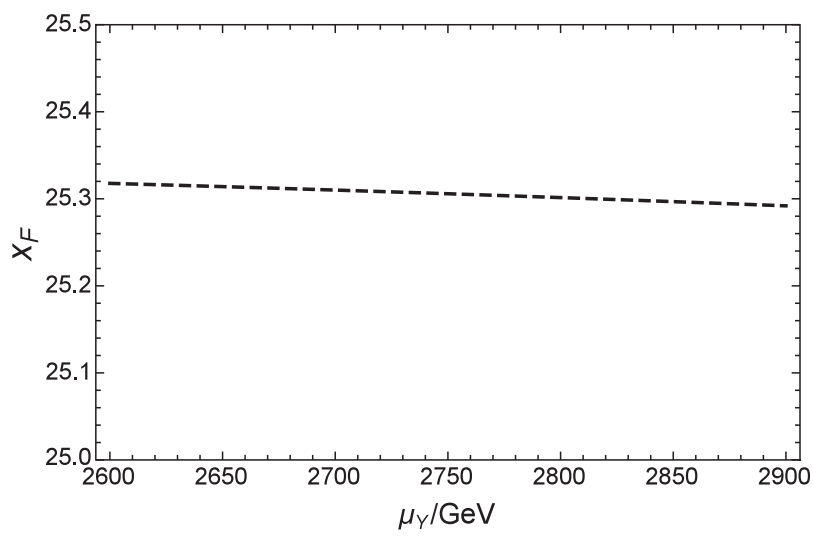

FIG. 5. The relic density and $x_{F}$ versus $\mu_{Y}$ for $\tilde{Y}$. 

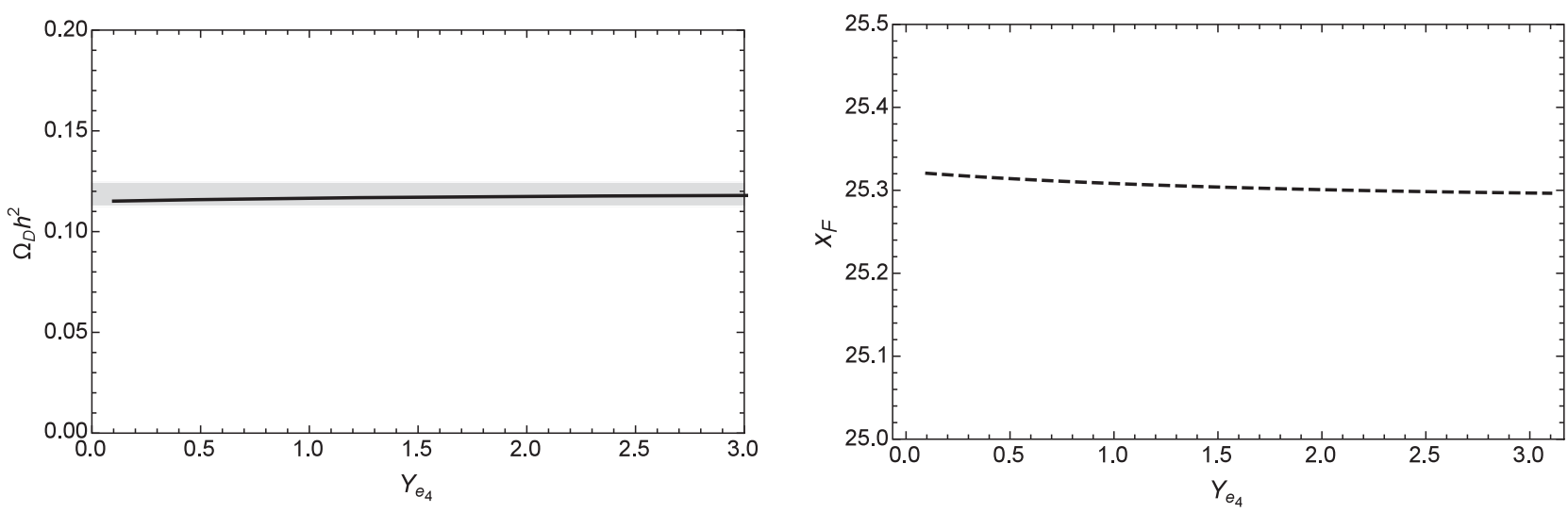

FIG. 6. The relic density and $x_{F}$ versus $Y_{e 4}$ for $\tilde{Y}$.

The parameters that are repeated with the subsection A are not listed, and they can all be found in Eq. (24). In Fig. 5, we study effects from the new parameters $\mu_{Y}$ on our numerical results. We use $\mu_{Y}=m_{D}, \quad \lambda^{\prime}=0.7$, $Y_{e 4}=1.3, L_{m}=0.8$. The reasonable range of $\mu_{Y}$ is 2650-2850 GeV. The Fig. 5 shows that larger $\mu_{Y}$ can lead to larger $\Omega_{D} h^{2}$, at the same time $\langle\sigma v\rangle$ decreases. The curve trend of $x_{F}$ is opposite to that of $\Omega_{D} h^{2}$.

In Fig. 6, taking $\lambda^{\prime}=1, \quad L_{m}=0.88, \quad \mu_{Y}=m_{D}=$ $2700 \mathrm{GeV}$, we plot the $\Omega_{D} h^{2}$ and $x_{F}$ versus $Y_{e 4} . Y_{e 4}$ is the Yukawa coupling constant that can influence the mass matrix of exotic slepton. When $Y_{e 4}$ gradually becomes larger, $\Omega_{D} h^{2}$ also increases accordingly. $x_{F}$ is limited to 24.6-24.8, with negligible change in the scope of meeting the relic density boundaries of dark matter. In general, both the dashed line and the solid line are flat and stable in the Fig. 5 and Fig. 6. The reason is that $m_{D}$ is taken as a fixed value in this figure.

\section{DISCUSSION AND CONCLUSION}

The extension of the BLMSSM is obtained by adding exotic superfields $\phi_{N L}, \varphi_{N L}, Y$ and $Y^{\prime}$ that can make the exotic leptons heavy and unstable. We call this new model as EBLMSSM, where we can deduce the mass matrices of particles and the couplings. The spinor $\tilde{Y}$ and the mixing of superfields $Y, Y^{\prime}$ are all new terms beyond BLMSSM. Furthermore, the exotic sleptons $\left(\tilde{L}^{\prime}\right)$ and exotic sneutrinos $\left(\tilde{N}^{\prime}\right)$ of generations 4 and 5 mix. Comparing with
BLMSSM the above enriches the content of lepton physics and dark matter physics.

We choose the lightest $\chi_{L}^{0}$ and $\tilde{Y}$ as dark matter candidates due to that they are consistent with the characteristics of cold dark matter. Then we research the relic density of $\chi_{L}^{0}$ and $\tilde{Y}$. In rational parameter space, $\Omega_{D} h^{2}$ can match the experiment bounds. Based on experimental data we can confine the sensitive parameters. The EBLMSSM inevitably will be a feature of many new physics models beyond the standard model. We believe the results in this paper maybe useful in the study of such models and the corresponding cosmological consequences.

\section{ACKNOWLEDGMENTS}

We are very grateful to Wei Chao the professor of Beijing Normal University, for giving us some useful discussions. This work is supported by National Natural Science Foundation of China (NNSFC) (No. 11535002, No. 11605037, No. 11705045), the Natural Science Foundation of Hebei province with Grant No. A2016201010 and No. A2016201069, Post-graduate's Innovation Fund Project of Hebei Province (No. CXZZBS2019027), Hebei Key Lab of OpticElectronic Information and Materials, the midwest universities comprehensive strength promotion project and the youth top-notch talent support program of the Hebei Province.
[1] G. Bertone, D. Hooper, and J. Silk, Phys. Rep. 405, 279 (2005).

[2] E. Corbelli and P. Salucci, Mon. Not. R. Astron. Soc. 311, 441 (2000).

[3] F. Zwicky, Helv. Phys. 6, 110 (1933).

[4] E. Corbelli, Mon. Not. R. Astron. Soc. 342, 199 (2003).
[5] D. Clowe, M. Bradac, A. H. Gonzalez, M. Markevitch, S. W. Randall, C. Jones, and D. Zaritsky, Astrophys. J. 648, L109 (2006).

[6] A. N. Taylor, S. Dye, T. J. Broadhurst, N. Benitez, and E. van Kampen, Astrophys. J. 501, 539 (1998). 
[7] D. Walsh, R. F. Carswell, and R. J. Weymann, Nature (London) 279, 381 (1979).

[8] M. Bradac, S. W. Allen, T. Treu, H. Ebeling, R. Massey, R. G. Morris, A. von der Linden, and D. Applegate, Astrophys. J. 687, 959 (2008).

[9] S. Penny, C. Conselice, S. De Rijcke, and E. V. Held, Mon. Not. R. Astron. Soc. 393, 1054 (2009).

[10] P. A. R. Ade et al., Astron. Astrophys. 571, 1054 (2014). arXiv: 1508.03375

[11] R. H. Cyburt, Phys. Rev. D 70, 023505 (2004).

[12] M. Drees and M. M. Nojiri, Phys. Rev. D 47, 376 (1993).

[13] J. L. Feng, Annu. Rev. Astron. Astrophys. 48, 495 (2010).

[14] S.-M. Zhao, T.-F. Feng, G.-Z. Ning, J.-B. Chen, H.-B. Zhang, and X. X. Dong, Eur. Phys. J. C 78, 324 (2018).

[15] Particle Data Group, Chin. Phys. C 40, 100001 (2016).

[16] P. F. Perez and M. B. Wise, Phys. Rev. D 82, 011901 (2010).

[17] T. R. Dulaney and P.F. Perez, Phys. Rev. D 83, 023520 (2011).

[18] P. F. Perez, Phys. Lett. B 711, 353 (2012).

[19] J. M. Arnold and P. F. Perez, Phys. Rev. D 85, 115024 (2012).

[20] X.-X. Dong, S.-M. Zhao, H.-B. Zhang, and T.-F. Feng, Eur. Phys. J. C 79, 17 (2019).
[21] S.-M. Zhao, T.-F. Feng, H.-B. Zhang, B. Yan, and X.-J. Zhan, J. High Energy Phys. 11 (2014) 119.

[22] X.-X. Dong, S.-M. Zhao, H.-B. Zhang, F. Wang, and T.-F. Feng, Chin. Phys. C 40, 093103 (2016).

[23] S.-M. Zhao, T.-F. Feng, X.-X. Dong, H.-B. Zhang, G.-Z. Ning, and T. Guo, Nucl. Phys. B910, 225 (2016).

[24] J. L. Feng, Annu. Rev. Astron. Astrophys. 48, 495 (2010).

[25] S. Andreas and T. Hambye, J. Cosmol. Astropart. Phys. 10 (2008) 034.

[26] J. L. Feng and J. Kumar, Phys. Rev. Lett. 101, 231301 (2008).

[27] W. Chao and H.-K. Guo, J. Cosmol. Astropart. Phys. 09 (2017) 009.

[28] J. McDonald, Phys. Rev. D 50, 3637 (1994).

[29] G. Blanger and F. Boudjema, Comput. Phys. Commun. 192, 322 (2015).

[30] W. Chao and H.-K. Guo, Phys. Lett. B 782, 517 (2018).

[31] W. Chao, J. High Energy Phys. 04 (2017) 034.

[32] C. P. Burgess and M. Pospelov, Nucl. Phys. B619, 709 (2001).

[33] X.-G. He and T. Li, Phys. Rev. D 79, 023521 (2009).

[34] J. L. Feng and T. M. Konstantin, Phys. Lett. B 482, 388 (2000). 